\section{PHYSICS OF IMPREGNATION}

\section{J. Roy Nelson, Material Testing Laboratory mtl@njcc.com}

There appears to be some confusion as to how materials are impregnated or dried. Without getting into the exciting mathematical details best suited for persons who second language is Greek, I offer the following short explanation as to how materials are impregnated or dried.

If the object is porous, the porosity is described in terms of macropores (>50 nm), mesopores $(50-2 \mathrm{~nm})$, and micropores $(2-0.8 \mathrm{~nm})$. These three size classes of pores represent different flow mechanisms for the impregnation of porous materials. Solvent penetration of a non-porous particle by imbibing is similar mathematically to solvent penetration of micropores although the actual mechanism is quite different ${ }^{1}$.

a) Flow through large pores or macropores is by bulk diffusion. In this case, the pores are large compared to the mean free path of the solvent molecule. Flow is greater when the pore size is larger or more pressure is applied. The kinetics are described by unsteady-state version of Flick's second law of diffu$\operatorname{sion}^{2}$ since rate varies with the degree of filling.

b) Flow through mesopores is by Knudsen diffusion ${ }^{3}$. In this case, the pores are smaller than the mean free path of the molecule. Flow is greater when the pore size is larger but independent of applied pressure. Over a limited change of temperature both bulk and Knudsen diffusion are essentially independent of temperature.

c) Flow through micropores or imbibing is by activated diffusion. In this case, the solvent molecules interact with the pore surface or molecular structure of the sample. The flow into the micropores or swelling of the material is exponentially dependent on temperature. At room temperatures equilibrium can take weeks or months.

On a more practical note, impregnation of a porous sample is faster in a vacuum. This can be done with a freezing trap with a separable ground glass collection tube. By adding valves to both the side outlet (to vacuum) and center inlet tubes (to solvent) and a liquid to be added to the sample under vacuum. For casting, we usually just made a deep aluminum foil pan, added the sample and then the resin. Place in vacuum oven, evacuate very slowly to degas, and then heat.

1. Patrick Meares, Polymers, Structure and Bulk Properties, 1967

2. S.P. Nandi, "The Unsteady State Diffusion of Gases from Coals," Ph.D. Thesis, The Pennsylvania State University, 1964 3. F.S. Karn, R.A. Friedal, and A.G. Sharkey Jr., "Mechanism of Gas Flow Through Coal," Fuel, 54, 1975

\section{Dil and DiO Confocal Imaging of Chicken Embryos Wilfred F. Denetclaw \\ University of California, School of Medicine wtdenet@concentric.net}

In my research, I am interested in understanding cellular morphogenetic events in early somitic myogenesis in the chicken embryo, and much of this work involves viewing fluorescently labeled cells in embryonic tissues. For this work, I am using $0.2 \%$ (weight/volume in $100 \%$ tetraglycol (Sigma)) concentrations of dil $\left(1,1^{\prime}\right.$, di-octadecyl-3,3,3',3',-tetramethylindocarbocyanine perchlorate) and $\mathrm{diO}\left(3,3^{\prime}\right.$-dioctadecyloxacarbocyanine perchlorate) to focally label small areas (approximately 10 cells) of the epithelial somite known as the dermomyotome.

Dye delivery is by iontophoresis ( 3 second pulses at 100 $\mu \mathrm{A})$. DiO is first solubilized in absolute ethanol, and then diluted in $100 \%$ tetraglycol. After dye injection and overnight embryo growth, embryos are removed from the egg, cleaned free of

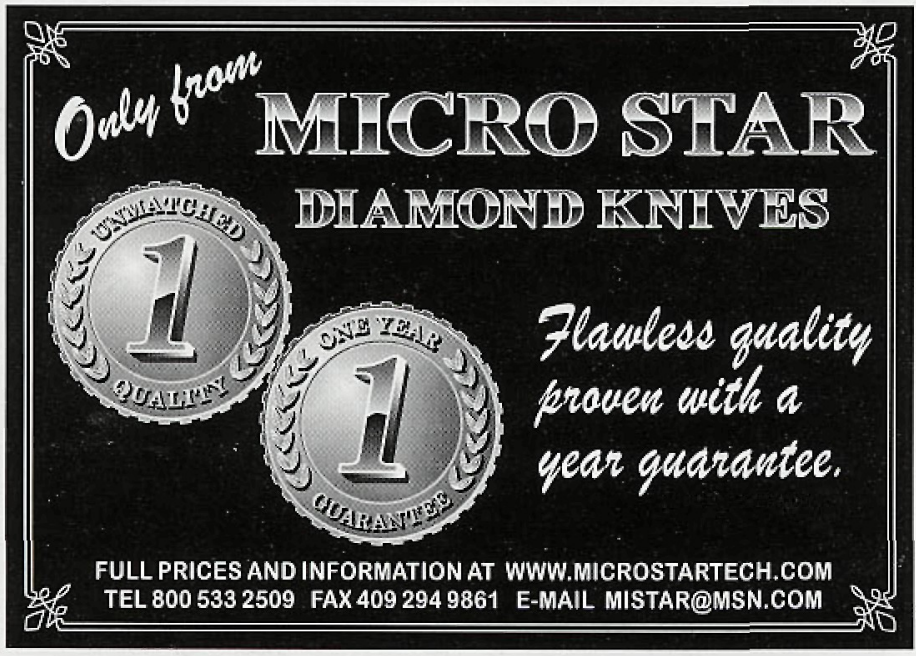

extra-embryonic tissues and fixed in $4 \%$ formaldehyde (in distilled water) for 4 hours at room temperature. The embryos are then washed in PBS and processed for microscopy or are stored at $4^{\circ}$ C.

For confocal imaging, embryos are bisected down the length of the neural tube, and transverse cuts are made several somites cranial and caudal to the injected somite, isolating a small tissue piece.

For each specimen, a custom chamber is easily made by layering 2-5 strips of electrical tape on a clean glass slide (number of layers depends on size of tissue). A small chamber is cut-out with a razor blade and the chamber is filled with phosphate buffered saline (PBS). The tissue is then placed in the chamber and positioned with forceps. A coverslip is placed over the tissue and nail polish is used to seal the slide. The electrical tape chamber provides a fast and convenient way to view tissue material with minimal distortion for microscopy.

For confocal imaging on a Nikon PCM2000, diO and dil labels are excited with appropriate argon ion (lower power setting, 488 $\mathrm{nm}$ filter) and green HeNe lasers and fluorescence is captured through FITC or Rhodamine emission filters, respectively. For mounting media, my experience is that dil and diO are highly stable and do not produce noticeable bleaching. However, with antibody labeled tissue, a mounting medium with $2 \%$ n-propyl-gallate (Sigma), mixed 1:1 in PBS:glycerol, $\mathrm{pH} 7.4$ in the electrical tape chamber is used to reduce bleaching.

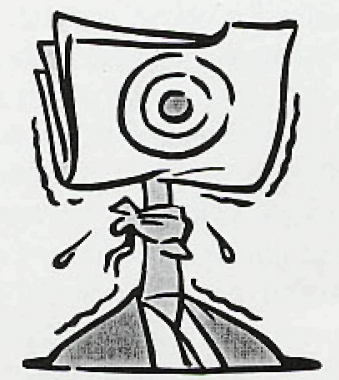

